Para enlazar con este artículo / To link to this article:

http://dx.doi.org/10.14198/fem.2017.29.12

Para citar este artículo / To cite this article:

Pastor Gosálbez, María Inmaculada, Paloma Pontón Merino y Ana Acosta Sarmiento. «El acceso a la carrera investigadora desde la perspectiva de género ipunto de inflexión?». En Marcos Jesús Iglesias Martínez e Inés Lozano Cabezas (coords.), La (in) visibilidad de las mujeres en la Educación Superior: retos y desafios en la Academia. Feminismo/s, 29 (junio 2017): 299-327, DOI: 10.14198/fem.2017.29.12

\title{
EL ACCESO A LA CARRERA INVESTIGADORA DESDE LA PERSPECTIVA DE GÉNERO ¿PUNTO DE INFLEXIÓN?
}

\section{THE ACCESS TO THE RESEARCH CAREER FROM A GENDER PERSPECTIVE. A TURNING POINT?}

\author{
María Inmaculada PASTOR GOSÁLBEZ \\ inma.pastor@urv.cat \\ orcid.org/0000-0003-4913-0722 \\ PALOMA PONTÓN MERINO \\ paloma.ponton@urv.cat \\ orcid.org/0000-0001-6929-8288 \\ ANA ACOSTA SARMIENTO \\ anacosta08@gmail.com \\ orcid.org/0000-0001-6847-3179 \\ Universitat Rovira i Virgili
}

\section{Resumen}

Las becas pre y postdoctorales son, actualmente, las vías de acceso principales a la carrera académica en el sistema universitario español. Diagnosticar los factores que intervienen en el acceso, desarrollo y mantenimiento diferencial entre mujeres y hombres es un requisito indispensable para la implementación de medidas dirigidas a lograr la igualdad en el trabajo. Así pues, en esta investigación, ponemos la atención en estas posiciones iniciales de la carrera académica. El objetivo perseguido es conocer las condiciones de trabajo y dinámicas que se dan, por mujeres y hombres, durante los primeros años de incorporación del personal docente e investigador en formación, a la Universidad. El estudio se ha basado en una encuesta y entrevista en profundidad a una muestra del Personal Investigador en Formación (PIF) de la Universitat Rovira i Virgili (URV). Los datos indican que existen diferencias en las condiciones de trabajo 
así como en las motivaciones, cargas docentes y expectativas que pueden condicionar el desarrollo diferenciado por género de la carrera investigadora.

Palabras clave: género, carrera académica, Universidad, desigualdades

\begin{abstract}
Pre and postdoctoral fellowships are currently the main access routes to the academic career in the Spanish university system. Diagnosing the factors involved in access, development and differential maintenance between women and men is an indispensable requirement for the implementation of measures aimed at achieving equality at work. Thus, in this research, we focus on these initial positions of the academic career. The objective is to know the working conditions and dynamics that are given by women and men during the first years of incorporation of teaching staff and researcher in training to the university. The study was based on a survey and in-depth interview with a sample of Research Staff in Training (PIF) of the Universitat Rovira i Virgili (URV). The data indicate that there are differences in the working conditions as well as in the motivations, teaching loads and expectations that can condition the differentiated development by gender of the research career.
\end{abstract}

Keywords: gender, academic career, university, inequalities 


\section{INTRODUCCIÓN: LOS DESEQUILIBRIOS DE GÉNERO EN LA UNIVERSIDAD ESPAÑOLA}

Diferentes estudios recientes (Sánchez de Madariaga; Sánchez de Madariaga et al. 2011; Generalitat de Catalunya; Guil) y anteriores (Pérez Sedeño et al.; García de León y García de Cortázar; Izquierdo 2004; Izquierdo 2008; Pastor) han puesto de manifiesto la pérdida de mujeres a lo largo de las diferentes etapas de la trayectoria investigadora y académica: en las primeras etapas representan más de la mitad del conjunto de investigadores pero su peso se va reduciendo hasta llegar a ser sólo el $20 \%$ en las categorías superiores. Aunque hoy en día las mujeres son mayoría entre el alumnado universitario y terminan sus estudios con expedientes académicos mejores, en promedio, que los de sus compañeros, ciertos ámbitos de conocimiento se resisten todavía a la participación femenina. Es el caso de las ingenierías y algunas ciencias experimentales donde la presencia de mujeres es inferior al 30\%. No es menos extremo por conocido el hecho de que en áreas como la Pediatría o la Ginecología y Obstetricia siga sin haber una sola catedrática. En los escalones más altos de la carrera científica y empresarial, el número de mujeres está prácticamente estancado y no refleja en absoluto el potencial de mujeres preparadas, con experiencia y méritos suficientes para acceder a estos puestos. Datos de diversas fuentes (Eurostat, ME, MICINN, INE, CSIC) llevan años demostrando empíricamente el desequilibrio que existe en la proporción, «seniority» y distribución de mujeres y hombres en las diversas ramas y categorías profesionales en el ámbito de la Universidad pública española y en los Organismos Públicos de Investigación.

En el marco español, el Libro Blanco. Situación de las mujeres en la ciencia española (Sánchez de Madariaga et. al. 2011), así como los sucesivos informes del informe del Ministerio de Educación, Cultura y Deporte (Datos y Cifras del Sistema Universitario Español) muestran datos para el conjunto de las universidades españolas y el análisis de las desigualdades por razón de género en el sistema universitario español. Estos documentos demuestran un primer hecho que consideramos relevante: la feminización del alumnado universitario. Para el curso 2013-2014, el 54\% del total de alumnado universitario, fueron mujeres. Este porcentaje sube ligeramente entre la población graduada $(57,3 \%)$. 
También es superior la proporción de mujeres entre el alumnado de máster $(53,7 \%)$. Sin embargo, cuando analizamos los datos por ámbitos de conocimiento, los datos se empiezan a desequilibrarse. Se observa una proporción de hombres muy superior a la de mujeres, en la rama de ingenierías y arquitectura $(74,1 \%)$; al tiempo que las mujeres tienen una presencia muy superior en la rama de ciencias de la salud $(70,7 \%)$.

A pesar de estos datos relativos a alumnado, la feminización de la Universidad no afecta al personal docente e investigador (PDI), ya que las mujeres docentes e investigadoras en España representan una media del 39,9\%. Esta cifra ha variado poco en los últimos veinte años: ya en el curso 1993-94 el porcentaje de mujeres PDI era del 31,09\% (Sánchez de Madariaga et. al., 2011). Del mismo modo que ocurre con el alumnado, el PDI está desigualmente distribuido por sexo según el ámbito de conocimiento, con la diferencia de que los hombres son mayoría en todos los ámbitos. En ingeniería y arquitectura representan un $79,4 \%$; un $55,8 \%$ en ciencias sociales y jurídicas; un $52,4 \%$ en artes y humanidades; un 55,8\% en ciencias de la salud; y un $61,6 \%$ en ciencias.

Si nos fijamos en el caso de las catedráticas, el porcentaje de mujeres se reduce al 20,7\% (según los datos del Ministerio del MECD para el curso 2013-2014). A pesar de la mejora en la cifra en los últimos años (en el curso 2004-2005, el primero en que el MECD publica esta estadística, las mujeres representaban el 13,8\% del total de catedráticos), la presencia de las mujeres en las cátedras sigue siendo muy baja. También lo es en otras posiciones, como las catedráticas de escuela universitaria $(29,3 \%)$.

El impulso, en el ámbito académico, a la aplicación de medidas para eliminar las desigualdades por razón de género ha venido de la mano de diferentes leyes e iniciativas políticas. En concreto, en el ámbito español se han tomado medidas y se han desarrollado acciones para mejorar la situación de las mujeres en la ciencia. Cabe destacar las siguientes leyes:

- Ley Orgánica 3/2007, de 22 de marzo, para la igualdad efectiva de mujeres y hombres, que fue publicada en el BOE n. ${ }^{\circ} 71$ de 23/3/2007 en páginas 12611 a 12645. Esta Ley, en su artículo 25, dedicado a la igualdad en la Educación Superior, apunta que las «Administraciones públicas en el ejercicio de sus respectivas competencias fomentarán la enseñanza y la investigación sobre el significado y alcance de la igualdad entre mujeres y hombres». Además se promueve la inclusión, en los planes de estudio en que proceda, de enseñanzas en materia de igualdad entre mujeres y hombres así como la creación de postgrados 
específicos y la realización de estudios e investigaciones especializadas en la materia.

- Ley Orgánica 4/2007, de 12 de abril, por la que se modifica la Ley Orgánica 6/2001, de 21 de diciembre, de Universidades publicada en el BOE» núm. 89, de 13 de abril de 2007, páginas 16241 a 16260, que en su Disposición adicional duodécima «Unidades de igualdad», define que «las universidades contarán entre sus estructuras de organización con unidades de igualdad para el desarrollo de las funciones relacionadas con el principio de igualdad entre mujeres y hombres».

- La Ley 14/2011 de la Ciencia, la Tecnología y la Innovación, publicada en el BOE num.131 de 2 de junio de 2011, páginas 54387 a 54455, en su disposición adicional decimotercera dedicada al desarrollo de la perspectiva de género, apunta que la perspectiva de género se promoverá como una categoría transversal en la investigación científica y técnica, que debe ser tenida en cuenta en todos los aspectos del proceso para garantizar la igualdad efectiva entre hombres y mujeres. Además, se establecen medidas concretas para la igualdad en este ámbito.

Por su parte, las instituciones europeas han mostrado su compromiso, también en el ámbito de la ciencia y la tecnología, con el desarrollo de actuaciones que promuevan la transformación de la realidad anteriormente descrita y hagan posible la igualdad efectiva entre hombres y mujeres. Cabe destacar que desde 2012, la igualdad y la transversalidad de género en los programas de investigación es una de las cinco prioridades planteadas por el European Research Area (ERA) ${ }^{1}$. Por su parte, el programa Horizonte 2020 (H2020) recoge el compromiso de la Comisión Europea de alcanzar la igualdad entre hombres y mujeres y establece 4 objetivos ${ }^{2}$ específicos de trabajo para hacerla posible:

- Lograr la igualdad en los equipos de investigación.

- Alcanzar la igualdad en los diferentes niveles de toma de decisión.

- Integrar el análisis de género/sexo en el contenido de la investigación y la innovación.

- Lograr la igualdad en el monitoreo y la evaluación de los proyecto de investigación.

Para alcanzar la igualdad de género en todas sus dimensiones es imprescindible identificar los factores que pueden estar incidiendo en esta pérdida de talento

1. Ver: http://ec.europa.eu/research/era/index_en.htm (Consultada en 10-07-2017)

2. Ver: http://www.eshorizonte2020.es/mas-europa/ciencia-con-y-para-la-sociedad/igualdadde-genero-en-la-investigacion-y-la-innovacion (Consultada en 10-07-2017) 
femenino, especialmente, en las primeras etapas de la carrera académica. Así, desde hace años, diversas autoras han llevado a cabo estudios para identificar las causas de las persistentes desigualdades que mantienen a las mujeres alejadas de determinadas posiciones profesionales, aquellas con más carga de responsabilidad, decisión, liderazgo y poder. También son numerosos los informes que se han llevado a cabo en diferentes países para medir dicha desigualdad y cuantificar la pérdida de mujeres en el desarrollo de las carreras académicas ${ }^{3}$. Asimismo, cabe sumar el esfuerzo de las propias universidades que a través de sus estructuras de igualdad (unidades, observatorios, centros, oficinas), se han encargado de desarrollar las medidas necesarias que garanticen, de manera efectiva, la igualdad de oportunidades para mujeres y hombres.

En esta línea, podemos destacar el experimento realizado por MossRacusin y colaboradores, que demostró el sesgo de género hacia las mujeres en los departamentos de ciencia. Para este estudio, se pidió a una serie de investigadores que enviaran un mismo plan de estudios, en algunos casos con nombre masculino y en otros con nombre femenino, solicitando un puesto como gerente de laboratorio. Las evaluaciones fueron diferentes según el sexo del solicitante a pesar de tener los mismos méritos. Para los hombres, las evaluaciones fueron mejores, sus currículums estuvieron mejor valorados, y se les propusieron mejores salarios de base, y más posibilidades de formación $\mathrm{y}$ ascenso.

En España, sin embargo, no contamos con suficientes estudios que den cuenta de las dificultades académicas, laborales y organizativas que se encuentran hombres y mujeres en los primeros estadios del desarrollo de la trayectoria académica. Y concretamente se echan de menos estudios sobre el colectivo de becarios y becarias predoctorales que analicen sus condiciones de trabajo, sus expectativas profesionales y los mecanismos que empiezan a generar las desigualdades en este momento incipiente de la carrera académica.

Cuando se trata de encontrar las causas de la mayor expulsión de mujeres de la carrera académica se suelen exponer diferentes líneas argumentales. Una de ellas apunta que es el peso de la maternidad lo que fundamentalmente incide en que ellas abandonen la carrera profesional. Otras líneas argumentales apuntan a la menor acumulación, por parte de ellas, de méritos suficientes para mantener expectativas de éxito profesional. A nuestro entender ninguna de estas líneas ha mostrado suficientes evidencias -especialmente cuando se refiere al grupo de investigadores hombres y mujeres, más jóvenes- como para

3. En Pastor y Acosta (2016: 251) se puede ver una tabla resumen de los informes más destacados que se han realizado en diferentes países europeos en los últimos años. 
resultar irrefutables. Consideramos que existen otros factores que pueden tener más peso en la creación de desigualdades. Así pues, nuestra hipótesis general es que no hay grandes diferencias de actitud, aspiraciones o interés entre hombres y mujeres, sino que el funcionamiento organizativo con sesgo de género de las propias universidades es lo que acabará produciendo desigualdades entre hombres y mujeres. Este sesgo de género de las organizaciones, ha sido estudiado profusamente por autoras como Acker, Nicolson, Ely y Meyerson o Barberá et al. En esta línea hipotizamos que son los siguientes factores los que tendrán un mayor papel en ese sesgo: el peso de las redes masculinas que perjudica directamente a las mujeres (Méndez), una división sexual del trabajo académico que también las penaliza (García de León), una falta de transparencia en los criterios de selección y unos criterios poco meritocráticos (Lara; Sánchez de Madariaga, 2011). Además, los sesgos de género se manifiestan a nivel directo en la forma que se atribuyen las competencias científicas a los hombres y las mujeres. Existen estándares diferentes para valorar el desempeño académico y científico de los hombres y las mujeres en las universidades y los centros de investigación en el área de la docencia, la gestión y la investigación científica (Foschi). Todos estos factores no han estado, a nuestro entender suficientemente estudiados en las universidades españolas entre el colectivo que es objeto de nuestra reflexión, las becarias y los becarios predoctorales.

En este artículo se presenta un estudio centrado en una universidad y ofrece una aproximación a la realidad laboral y académica del colectivo citado; jóvenes que dan los primeros pasos en el desarrollo de la trayectoria académica y que van recibiendo un trato, una información, unos estímulos, unas recompensas y unos castigos diferenciados por género y que tienen un impacto importante sobre su carrera profesional en la Universidad. Asimismo, se ofrecen los resultados de una investigación centrada en los becarios y becarias de la URV pero también se presentan ideas y conocimientos fruto de investigaciones anteriores centradas en el estudio de las desigualdades de género en la universidad así como de las políticas de igualdad desarrolladas en ellas.

Este artículo representa una aproximación a un objeto de estudio que, a nuestro entender, requiere de un estudio más exhaustivo, incluyendo la lógica diacrónica para recoger los cambios generacionales y el análisis del potencial impacto de las leyes y las transformaciones que la universidad española está experimentando. 


\section{EL GÉNERO DE LAS BECAS EN LA UNIVERSIDAD}

Como se describió en el apartado anterior, a pesar de la importancia de las becas o ayudas predoctorales y postdoctorales para el inicio de la carrera investigadora en el ámbito universitario, existen pocos estudios dedicados a los mismos. Por otra parte, la propia diversidad de esta figura de investigador novel, ha llevado a que incluso normativamente se presente como un colectivo poco regulado, hasta la aprobación del Real Decreto 1326/2003 por el que se aprueba el Estatuto del becario de investigación. Tres años más tarde se aprobaría el Real Decreto 63/2006, que amplía el concepto de becario de investigación y lo enmarca, fijando su relación jurídica contractual de acuerdo al marco vigente:

Tienen la condición de personal investigador en formación aquellos graduados universitarios que sean beneficiarios de programas de ayuda dirigidos al desarrollo de actividades de formación y especialización científica y técnica a través, como mínimo, de los correspondientes estudios oficiales de doctorado, sin perjuicio de las especialidades previstas en la Ley 44/2003, de 21 de noviembre, de ordenación de las profesiones sanitarias.

En cuanto a los estudios recientes que han dedicado atención al colectivo de becarios cabe destacar el Libro blanco. Situación de las mujeres en la Ciencia Española (2011). En esta publicación se dedica un apartado a analizar específicamente las diferencias por género en las ayudas públicas a la formación de postgrado y de postdoctorado en España. El estudio ofrece datos tanto de la concesión de becas como de su solicitud y su evolución entre los años 2002 y 2009, si bien en algunas modalidades de beca también se ofrece información de años anteriores. Estos datos nos permiten contar con un punto de partida para situar nuestra aproximación. Sin embargo, mantenemos la necesidad de ampliar este tipo de estudios recogiendo tanto la opinión de los sujetos en relación a algunos aspectos muy relevantes de sus opciones de carrera académica, como de las condiciones de trabajo y su vivencia, como hombres o como mujeres, del funcionamiento de la Universidad. Esta información también arrojaría luz sobre un proceso que diversos expertos apuntan como clave en la Universidad española en las últimas dos décadas, nos referimos a la precarización de una parte de las plantillas docentes y su impacto en la organización.

Las principales conclusiones del Libro blanco son:

- En el período 1998-2007 se mantiene la elevada sobrerrepresentación de universitarias en educación, ciencias de la salud y humanidades; en contraposición con la infrarrepresentación de este colectivo en 
ingenierías y ciencias y matemática. Es de destacar que en el período analizado se observaron sutiles cambios a favor de carreras más equilibradas.

- Se puede apreciar mayor presencia de mujeres como becarias predoctorales que posdoctorales. Excepto en el programa Fulbright, tanto predoctoral como posdoctoral, en el cual no ha habido incrementos mantenidos en la participación de mujeres.

- En el programa de becas predoctorales, a pesar de ser las que cuentan con una mayor representación de mujeres, se encuentran un $10 \%$ por debajo de proporción de mujeres calificadas para disfrutar de esta modalidad de beca.

- Los programas Juan de la Cierva y Ramón y Cajal cuentan con cifras notablemente negativas para las mujeres, encontrando incluso retrocesos respecto a sus primeros programas.

Más allá de estos datos, no se han encontrado otros estudios que abarquen a la totalidad de la Universidad española, por lo que, los datos que expondremos a continuación, a pesar de abarcar el universo de la URV, pueden considerarse relevantes a modo de aproximación a esta temática.

\subsection{Becarios y becarias en la URV}

En la URV se reproducen, aunque amortiguados, estos desequilibrios. El alumnado que se matricula en la URV es mayoritariamente femenino (59\%). No obstante, encontramos áreas de conocimiento absolutamente polarizadas. Por ejemplo, para el curso 2014-2015, sólo 18 chicas se matricularon en el Grado de Ingeniería Eléctrica (donde se matricularon 208 chicos) y 28 al Grado de Ingeniería Mecánica (donde se matricularon 261 chicos). Por el contrario, 149 chicos se matricularon en el Grado de Enfermería (donde lo hicieron 648 chicas) y 45 chicos se matricularon en el Grado de Educación Infantil (donde lo hicieron 781 chicas). Si miramos las matrículas de Máster y Postgrado, vemos que ellas siguen siendo mayoría, con un 53\%. Pero también encontramos diferencias por áreas de conocimiento. Por ejemplo, sólo 1 chica se matriculó en el Máster en Ingeniería industrial (lo hicieron 49 chicos) y 9 chicos se matricularon en el Máster en Investigación en Ciencias de la Enfermería (donde lo hicieron 50 chicas). En los estudios de Doctorado, las chicas siguen siendo mayoría (un 56\%). La balanza se empieza a decantar en las tesis leídas: para el curso 2013-14, las tesis leídas por mujeres representaron el 48\% del total. A partir de este momento, la realización de la tesis, el número de mujeres 
comienza a decrecer hasta llegar a las categorías más altas: las mujeres catedráticas representan sólo el $28 \%$ de las cátedras. Así pues, pasamos de una mayoría femenina (59\%) en las fases iniciales de la carrera académica a una minoría en las posiciones más altas (28\%). A partir de esta breve diagnosis inicial, vamos a profundizar en las dinámicas y barreras de la universidad que están generando este desequilibrio.

En la URV, en diciembre de 2014, 279 personas formaban parte del colectivo «Personal Investigador en Formación» (PIF). Según la clasificación del Servicio de Recursos (SRH) de la universidad, existen 10 categorías de PIF. Las mujeres representan el 53,8\% (150) del PIF, mientras que los hombres son el $46,2 \%$ (129). Si analizamos las categorías según sexo obtenemos los siguientes datos (Tabla 1) ${ }^{4}$ :

Tabla 1. Personal Investigador en Formación según tipo de beca y sexo

\begin{tabular}{|l|l|l|}
\hline Tipo de PIF & Hombres & Mujeres \\
\hline 1 Ayudante Doctor / a & 33.3 & 66.7 \\
\hline 2 Beca Predoctoral MEC & 50 & 50 \\
\hline $\begin{array}{l}\text { 3 Beca Predoctoral } \\
\text { MEC-FPU-S.Social }\end{array}$ & 100 & 0 \\
\hline 4 PIF. MEC.FPI- contrato & 63.3 & 36.4 \\
\hline 5 PIF. MEC.FPU-contrato & 28.6 & 71.4 \\
\hline 6 PIF, & 42.9 & 57.1 \\
\hline 7 PIF. FPI, & 76.9 & 23.1 \\
\hline 8 PIF. FPU & 0 & 100 \\
\hline 9 PIF. URV & 48.9 & 51.1 \\
\hline 10 Personal Investigador Novel & 38.8 & 61.2 \\
\hline
\end{tabular}

Esta diferencia por tipo de beca no es especialmente significativa, ya que en la mayoría de casos, la modalidad de beca corresponde al año de doctorado en el que se encuentre que el PIF. Donde las diferencias sí son significativas es en la distribución del PIF por sexo y departamento. Cuando hacemos este análisis, observamos que existen departamentos altamente feminizados o masculinizados. En un extremo, tenemos departamentos como Ingeniería Electrónica, Eléctrica y Automática o Ingeniería Mecánica, muy masculinizados. En el otro extremo, tenemos departamentos como Bioquímica y Biotecnología o Medicina y Cirugía, muy feminizados. A la vez, en algunos departamentos se mantiene la paridad, como Química Analítica y Química Orgánica o en Ingeniería Química. Veamos estos datos en la Tabla 2.

4. Todas las tablas y figuras son de elaboración propia. Los datos relativos a la URV han sido proporcionados por el SRH de la misma universidad. 
Tabla 2. Personal Investigador en Formación según departamento y sexo

\begin{tabular}{|c|c|c|c|}
\hline Rama de conocimiento & Departamento & Hombres & Mujeres \\
\hline \multirow[t]{3}{*}{ Ciencias de la salud } & Ciencias médicas y básicas & 5 & 11 \\
\hline & Enfermería & 0 & 2 \\
\hline & Medicina y cirugía & 0 & 7 \\
\hline \multirow{9}{*}{$\begin{array}{|ll|}\begin{array}{l}\text { Ciencias } \\
\text { jurídicas }\end{array} & \text { sociales } \\
\end{array}$} & Antropología, trabajo social y filosofia & 1 & 5 \\
\hline & Derecho privado, procesal y financiero & 0 & 1 \\
\hline & Derecho público & 1 & 5 \\
\hline & Economía & 1 & 11 \\
\hline & Estudios de comunicación & 1 & 2 \\
\hline & Geografia & 4 & 8 \\
\hline & Gestión de empresas & 2 & 9 \\
\hline & Pedagogía & 2 & 3 \\
\hline & Psicología & 2 & 5 \\
\hline \multirow[t]{3}{*}{ Ciencias } & Química analítica y orgánica & 0 & 9 \\
\hline & Química física e inorgánica & 11 & 11 \\
\hline & Bioquímica y biotecnología & 3 & 25 \\
\hline \multirow[t]{5}{*}{ Ingeniería y arquitectura } & Ingeniería electrónica, eléctrica y automática & 20 & 5 \\
\hline & Ingeniería informática y matemática & 11 & 5 \\
\hline & Ingeniería mecánica & 14 & 5 \\
\hline & Ingeniería química & 18 & 19 \\
\hline & Arquitectura & 0 & 5 \\
\hline \multirow[t]{4}{*}{ Artes y humanidades } & Estudios ingleses y alemanes & 2 & 2 \\
\hline & Filología catalana & & \\
\hline & Filologías románicas & 0 & 1 \\
\hline & Historia e historia del arte & 4 & 4 \\
\hline
\end{tabular}

En cuanto a la edad del PIF de la URV, la mayoría se sitúan en el rango comprendido entre los 20 y los 29 años: un $71,3 \%$ de las mujeres, y un 63,6\% de los hombres.

\section{METODOLOGÍA}

Para la realización del presente estudio, en primer lugar, se han analizado todos los datos disponibles de la URV sobre PIF, datos que han sido proporcionados por el SRH de la propia universidad. Estos datos ofrecen información relevante pero insuficiente para conocer las condiciones de desarrollo profesionales de los becarios de manera que se nos presentó como imprescindible llevar a cabo una recogida de información específica para contrastar las hipótesis que, en tanto que investigadoras, hemos ido planteando en relación a este colectivo. Para ello se diseñó y envió una encuesta a todo el PIF de la universidad. Esta encuesta ha llegado a 279 personas (total PIF URV) y ha sido contestada por 
un $26,2 \%$ (de las cuales un $38,4 \%$ hombres y un $61,6 \%$ mujeres). La encuesta se dividió en 5 grandes bloques de preguntas:

- Bloque 1. Datos personales

- Bloque 2. Datos de la beca

- Bloque 3. Condiciones de trabajo

- Bloque 4. Vida cotidiana

- Bloque 5. Perspectivas de futuro

Finalmente, a todas las personas que contestaron la encuesta, se les propuso hacer una entrevista en profundidad. El nivel de participación en las entrevistas fue del $9,6 \%$, de las cuales un $28,6 \%$ hombres y un $71,4 \%$ de mujeres. El objetivo de las entrevistas era complementar y profundizar en los resultados obtenidos de la encuesta. Las entrevistas se organizaron a partir del mismo esquema de bloques de la encuesta. Se hizo hincapié en el Bloque 2 (Datos de la beca) para conocer mejor los detalles de la vía de acceso a la carrera académica y los motivos de optar a una beca; en el Bloque 3 (Condiciones de trabajo) para profundizar en el día a día de la carrera académica, las características y la valoración que hace el PIF de su trabajo; y en el Bloque 5 (Expectativas de futuro) para detectar las percepciones respecto al mantenimiento en la carrera académica. Es decir, a partir de la entrevista hemos podido obtener más información sobre el acceso, el desarrollo y el mantenimiento en la universidad. En los apartados que siguen presentamos el resultado y análisis de la encuesta y de las entrevistas.

Para la realización de este estudio hemos agrupado las 10 categorías con que trabajan SRH en 5 más categorías más funcionales, eliminando de la muestra la figura de Ayudante Doctora/a, por la poca representatividad (sólo 3 personas ocupan esta categoría). La recategorización queda como muestra la Tabla 3 .

Tabla 3. Recategorización

\begin{tabular}{|l|l|}
\hline Tipo de PIF & Recategorización \\
\hline 1 Ayudante Doctor / a & 5. Otras \\
\hline 2 Beca Predoctoral MEC & 1 . FPI \\
\hline 3 Beca Predoctoral MEC-FPU-S.Social & 2. FPU \\
\hline 4 PIF. MEC.FPI- contrato & 1. FPI \\
\hline 5 PIF. MEC.FPU-contrato & 2. FPU \\
\hline 6 PIF, AGAUR & 3. AGAUR \\
\hline 7 PIF. FPI, & 1. FPI \\
\hline 8 PIF. FPU & 2. FPU \\
\hline 9 PIF. URV & 2. FPU \\
\hline 10 Personal Investigador Novel & 4. URV \\
\hline
\end{tabular}




\section{IDENTIFICANDO LAS BARRERAS DE GÉNERO EN LAS ETAPAS INICIALES DE LA ACTIVIDAD INVESTIGADORA}

Las personas encuestadas son 28 hombres $(38,4 \%)$ y 45 mujeres $(61,6 \%)$. La muestra queda ligeramente desequilibrada respecto al universo, donde tenemos un $46,2 \%$ de hombres y un $53,8 \%$ de mujeres. En cuanto a la edad, en términos globales, el PIF encuestado se sitúa mayoritariamente $(65,8 \%)$ en el rango de edad 20-29 años. El siguiente rango de edad con mayor presencia es el de 30-39 (30,1\%). No han sido encontradas diferencias significativas por sexo en cuanto a la edad.

En cuanto a la universidad de procedencia (Tabla 4), el 63\% de los encuestados ha cursado los estudios previos al Doctorado en la misma URV. Si nos fijamos en los datos desagregados por sexo, vemos que las mujeres provienen en su mayoría de la misma universidad donde han cursado el máster o estudio que les ha dado acceso al Doctorado. En el caso de los hombres, la procedencia está más repartida entre URV, Universidades catalanas y Universidades españolas.

Tabla 4. Universidad de procedencia del PIF por sexo

\begin{tabular}{|c|c|c|c|c|}
\hline \multirow{2}{*}{$\begin{array}{l}\text { Universidad de } \\
\text { procedencia }\end{array}$} & \multicolumn{2}{|c|}{ Mujeres } & \multicolumn{2}{|c|}{ Hombres } \\
\hline & Total & $\%$ & Total & $\%$ \\
\hline URV & 32 & 72.7 & 13 & 46.4 \\
\hline Univ. catalana & 2 & 4.5 & 5 & 17.9 \\
\hline Univ. Española & 4 & 9.1 & 6 & 21.4 \\
\hline Univ. Europea & 2 & 4.5 & 1 & 3.6 \\
\hline Univ. no Europea & 4 & 9.1 & 3 & 10.7 \\
\hline Total & 44 & 100 & 28 & 100 \\
\hline
\end{tabular}

\subsection{Acceso: decidir optar a una beca predoctoral}

El PIF presentan diversas motivaciones para optar a una beca predoctoral, condicionadas por factores diferentes en cada caso. Sintetizando, los motivos que se hacen explícitos en las entrevistas son:

1. Para hacer el doctorado / Dedicarse a la investigación y docencia universitaria. Los argumentos dentro del primer grupo estarían ligados a la vocación, a diferencia de los del segundo grupo que serían argumentos más prácticos, donde la vocación sería más el efecto que la causa del inicio de la carrera académica.

2. Para tener un trabajo / Cambiar de trabajo / Consolidar una situación profesional. Los argumentos dentro de este grupo se sitúan más en el plano instrumental, en el que optar a hacer el doctorado es visto como 
una opción laboral más o un cambio respecto a la situación laboral presente.

Parece que los hombres se sitúan en el plano vocacional más que las mujeres. Es decir, la opción de los hombres de empezar la carrera académica es más activa, en el sentido que se inicia por iniciativa propia. Por lo tanto, se muestra un mayor grado de interés al inicio, y unas mejores expectativas futuras.

A partir de los resultados de la encuesta se puede apreciar que hay una diferencia significativa entre mujeres y hombres, a favor de ellas, en la modalidad de becas FPI, FPU y AGAUR, convocatorias mucho más competitivas que las becas URV, donde se concentran mayoritariamente los hombres (Tabla 5).

Tabla 5. Tipo de beca del PIF por sexo

\begin{tabular}{|l|l|l|l|l|}
\hline \multirow{2}{*}{ Tipo de beca } & Mujeres & Hombres \\
\cline { 2 - 5 } & Total & $\%$ & Total & $\%$ \\
\hline FPI & 5 & 11.4 & 3 & 10.7 \\
\hline FPU & 2 & 4.5 & 0 & 0 \\
\hline AGAUR & 12 & 27.3 & 5 & 17.9 \\
\hline URV & 22 & 50 & 17 & 60.7 \\
\hline Otras & 3 & 6.8 & 3 & 10.7 \\
\hline Total & 44 & 100 & 28 & 100 \\
\hline
\end{tabular}

En cuanto a la vía de conocimiento de las becas (Tabla 6), la mayoría de mujeres han conocido las becas a través de su director o directora de tesis, mientras que los hombres la han conocido a través de la universidad donde cursaron el máster. Es significativo que ninguna mujer ha conocido la beca a través del grupo de investigación, mientras que sí lo han hecho el 14,3\% de los hombres. Este dato sustenta la hipótesis del mayor uso que hacen los hombres de las redes de contactos personales.

Tabla 6. Medio de conocimiento de la beca predoctoral

\begin{tabular}{|l|l|l|l|l|}
\hline \multirow{2}{*}{ Fuente de información } & \multicolumn{3}{|l|}{ Mujeres } & \multicolumn{3}{l|}{ Hombres } \\
\cline { 2 - 5 } & Total & $\%$ & Total & $\%$ \\
\hline Universidad donde cursaron el Máster & 12 & 27.3 & 11 & 39.3 \\
\hline Director/a de tesis & 19 & 43.2 & 8 & 28.6 \\
\hline Entidad financiadora de la beca & 8 & 18.2 & 5 & 17.8 \\
\hline Grupo de investigación & 0 & 0 & 4 & 14.3 \\
\hline Otras & 5 & 11.4 & 0 & 0 \\
\hline Total & 44 & 100 & 28 & 100 \\
\hline
\end{tabular}


Si nos fijamos en los departamentos a los que están adscritos los encuestados, la distribución es la siguiente (Figura 1):

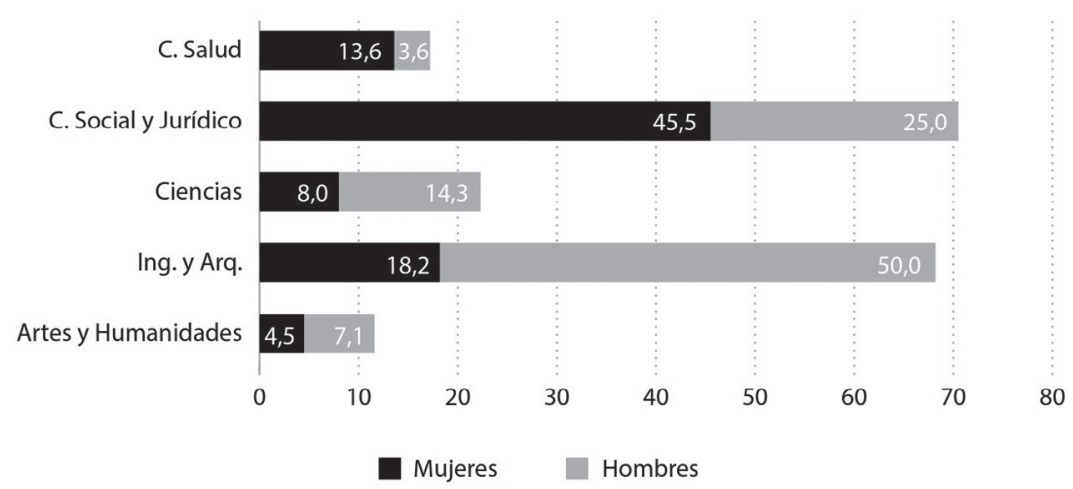

Figura 1. Distribución de encuestados por sexo y ámbito de conocimiento (\%)

\subsection{Desarrollo: características y valoración del trabajo}

En este bloque se quiso conocer cuáles son las condiciones de trabajo del PIF en la universidad. Concretamente, los pedimos información sobre su carga docente, sobre su participación en grupos de investigación y proyectos de investigación ajenos a la tesis y, finalmente, sobre si han realizado o tienen previsto hacerlo, una estancia de investigación superior a 3 meses. Es de destacar, en este sentido, lo que establece el real Decreto 63/2006, anteriormente citado:

El personal investigador en formación que desarrolle su actividad en una universidad podrá colaborar en tareas docentes, dentro de los límites que se establezcan en la correspondiente convocatoria, sin que en ningún caso pueda desvirtuarse la finalidad investigadora y formativa de las becas. En todo caso, no se le podrá atribuir obligaciones docentes superiores a 60 horas anuales.

Los datos recogidos nos muestran diferencias significativas en cuanto a carga docente (Tabla 7). Los resultados nos dicen que hay más hombres que mujeres sin carga docente. Además, si nos fijamos en cuántos créditos está asumiendo cada colectivo, encontramos que un 40,9\% de hombres tienen menos de 3 créditos, mientras que las mujeres que imparten menos de 3 créditos son el $26,3 \%$. Es decir, las mujeres asumen más docencia que los hombres e imparten más créditos. Esta tendencia suele extenderse a lo largo de la carrera académica, encontrando en el colectivo de mujeres una mayor carga de créditos. 
Tabla 7. Carga docente del PIF

\begin{tabular}{|l|l|l|l|l|}
\hline \multirow{2}{*}{ Fuente de información } & Mujeres & \multicolumn{3}{l|}{ Hombres } \\
\cline { 2 - 5 } & Total & $\%$ & Total & $\%$ \\
\hline No imparten docencia & 6 & 13.6 & 6 & 21.4 \\
\hline Imparten docencia & 38 & 86.4 & 22 & 78.6 \\
\hline$-\quad$ Menos de 3 créditos & 10 & 26.3 & 9 & 40.9 \\
\hline$-\quad$ Entre 3 y 6 créditos & 28 & 73.7 & 12 & 54.5 \\
\hline$-\quad$ Más de 6 créditos & 0 & 0 & 1 & 4.5 \\
\hline Total & 44 & 100 & 28 & 100 \\
\hline
\end{tabular}

En cambio, en cuanto a participación en grupos de investigación y en proyectos (Figura 2), no encontramos diferencias significativas. La amplia mayoría de PIF participa en grupos de investigación y aproximadamente la mitad en proyectos de investigación ajenos a su tesis. Hay que apuntar que estas dinámicas responden a una política científica general de la Universidad española en las que se está impulsando la participación en grupos de investigación para todo el conjunto del PDI. Es decir, no se debe a una dinámica específica de departamento o rama de conocimiento sino que las propias políticas de financiación de la investigación han impulsado la incorporación del personal en formación

\section{Participación en grupos de investigación}
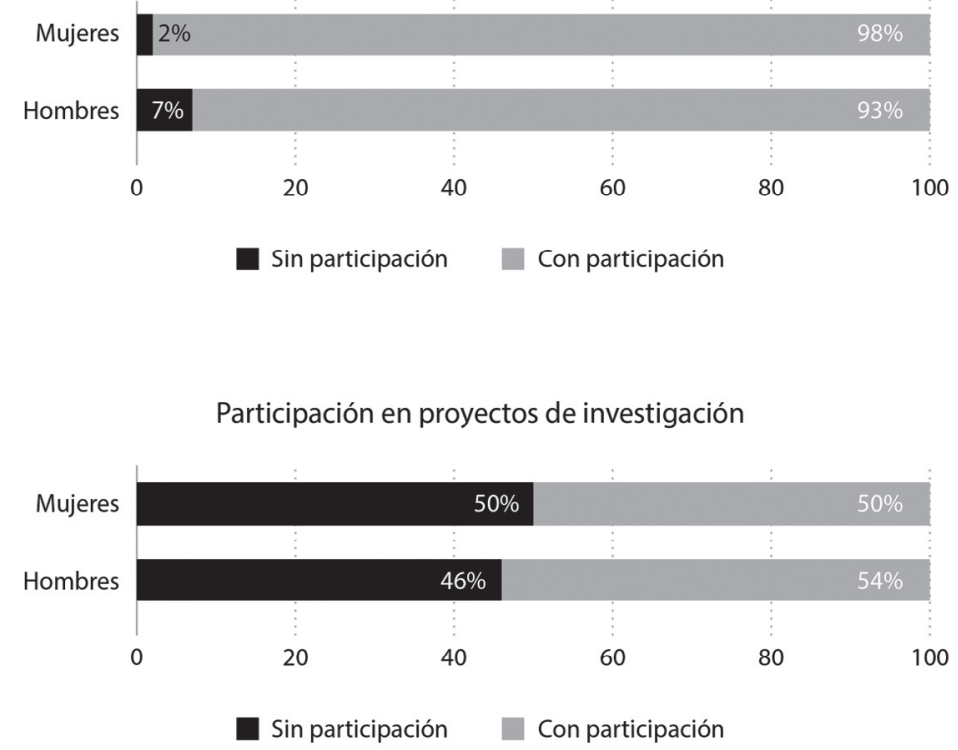

Figura 2. Participación del PIF en grupos y proyectos de investigación 
en los grupos y proyectos de investigación. Ponemos esto de manifiesto porque muestra una de nuestras líneas argumentales a la hora de explicar el impacto de las medidas a favor de la igualdad de género: cuanto más transparentes y meritocráticos sean los procesos de financiación de los grupos de investigación, los proyectos y las propias estructuras de las universidades, menos espacio habrá para la desigualdad de género. Línea argumental que está en consonancia con lo expuesto por otras investigadoras como Wennerås y Wold, en su, ya clásico, estudio sobre nepotismo y sesgos sexistas en la selección de investigadores.

En cuanto a las estancias de investigación en el extranjero (Figura 3), tampoco encontramos diferencias significativas: el 84\% de mujeres y el $85 \%$ de hombres han hecho o tienen previsto hacer una estancia. Este dato incide en nuestras hipótesis: las diferencias entre hombres y mujeres son fruto, sobre todo, de las dinámicas organizativas de las propias universidades y no están generadas por una manera de ser diferente de hombres y mujeres. Otra cosa será lo que finalmente puedan o no puedan hacer hombres y mujeres según su situación personal, como por ejemplo la carga de trabajo doméstico o las cargas familiares.

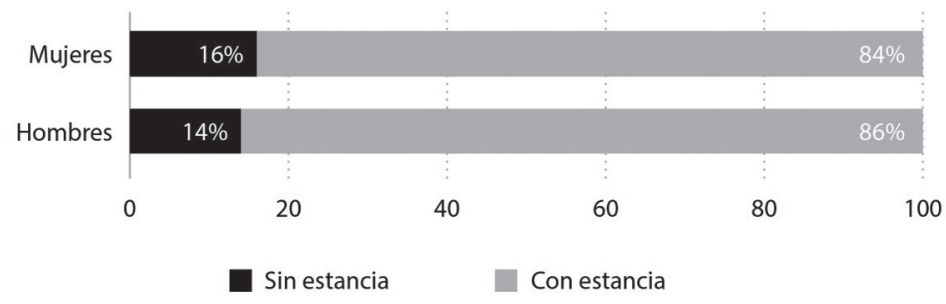

Figura 3. Estancias de investigación del PIF

En este apartado prestamos especial atención a una cuestión central en la actividad académica del PIF: la compaginación de la tesis doctoral con su trabajo cotidiano en el grupo de investigación y departamento en el que están adscritos. A partir de las respuestas, obtenemos la valoración que hace el PIF de su trabajo y de su experiencia personal como becaria o becario. En este apartado también profundizamos en la estancia de investigación en el extranjero, como una de las metas que la mayor parte del PIF de la URV pretende alcanzar (un $85 \%$ han hecho o tienen previsto realizar una estancia de investigación).

Como se observó anteriormente, la mayoría del PIF realiza alguna tarea de apoyo a la docencia, como impartir clases teóricas o prácticas, realizar sustituciones o colaborar en labores de vigilancia de exámenes. En cuanto a la 
investigación, a parte de la tesis se realizan también otras actividades, como la ayuda en proyectos o experimentos de otros investigadores/as, colaboración con el grupo de investigación, asistencia a congresos, etc. En este sentido, no parece que los entrevistados muestran diferencias por sexo, y no se les atribuyen cargas de trabajo diferentes.

En cuanto al trabajo cotidiano, nos centramos en cómo la carga docente y la participación en proyectos de investigación ajenos a la temática de la tesis (cuando los hay) impactan en la elaboración de la tesis doctoral. Estos son los dos factores que identifican los entrevistados como más problemáticos para realizar la tesis doctoral, ya que a menudo hacen detener por completo, durante un tiempo determinado, su realización. Vemos que, además de las tareas de investigación y docencia, en algunos casos hay que sumar cargas de actividades de gestión.

La docencia y la participación en proyectos de investigación ajenos a la tesis, sin embargo, también pueden ser vistas como positivos, en el sentido que, aunque supongan más carga de trabajo, instrumentalmente es positivo para el currículo académico. El otro punto de vista es el de las personas que no tienen asignada docencia ni colaboran en proyectos de investigación ajenos a su tesis doctoral. En este caso, la situación es vista como óptima, ya que el tiempo de dedicación exclusiva a la tesis aumenta.

En cuanto a la estancia de investigación, la mayoría de los entrevistados han hecho o tienen previsto realizarla, bien sea porque optan al título de doctores/ as con mención internacional, bien sea para vivir la experiencia de trabajar un tiempo en otra universidad. Desde la perspectiva de género debemos destacar, como bien apuntan otras investigaciones (por ejemplo, Mora, 2007), la incidencia de la maternidad y la conciliación de la vida personal, laboral y familiar en la decisión de realizar estancias de investigación, que afecta negativamente a las mujeres. Entre las personas entrevistadas, tenemos el caso de $G$, que ilustra esta dificultad:

No haré ninguna estancia, es imposible, tengo dos niños pequeños. Por las mismas condiciones económicas de la beca sería imposible. No me lo planteo. Como mucho, pensaría en hacer alguna estancia cerca, en Barcelona, en Lleida, pero no más lejos. (G. Mujer. 42 años)

\subsection{Vida cotidiana}

El bloque 4 tiene por objetivo profundizar en cómo es la vida cotidiana del PIF, especialmente en cuanto a la composición del hogar, a las fuentes de ingresos del hogar y la dedicación al trabajo reproductivo (trabajo doméstico, de atención y cuidado). 
En cuanto al tipo de hogar (Tabla 8), encontramos que las mujeres viven mayoritariamente con su pareja $(43,2 \%)$, mientras que los hombres viven mayoritariamente solos o con compañeros/as de piso (46,4\%). Para el resto de tipología de hogar, no hay diferencias significativas

Tabla 8. Tipo de hogar (convivencia)

\begin{tabular}{|l|l|l|l|l|}
\hline \multirow{2}{*}{ Fuente de información } & Mujeres & \multicolumn{3}{l|}{ Hombres } \\
\cline { 2 - 5 } & Total & $\%$ & Total & $\%$ \\
\hline Con los padres & 5 & 11.4 & 4 & 14.4 \\
\hline Solo/a o con compañeros de piso & 15 & 34.1 & 13 & 46.4 \\
\hline Con la pareja & 19 & 43.2 & 8 & 28.6 \\
\hline Solo/a + hijos & 0 & 0 & 0 & 0 \\
\hline Pareja e hijos & 4 & 9.1 & 3 & 10.7 \\
\hline Otros & 1 & 2.3 & 0 & 0 \\
\hline Total & 44 & 100 & 28 & 100 \\
\hline
\end{tabular}

En la fuente principal de ingresos del hogar encontramos diferencias entre mujeres y hombres: los hombres muestran una mayor dependencia de los padres ( $14,3 \%$, frente al $6,8 \%$ de las mujeres) y de su pareja (10,7\%, frente al $6,8 \%$ de las mujeres). En todo caso, tanto para hombres como para mujeres, la fuente principal de ingresos del hogar es uno/a mismo/a. En el cuidado y atención a personas dependientes (Tabla 9), encontramos que hay más mujeres que hombres que asumen esta responsabilidad, si bien en ambos colectivos el porcentaje de personas que tienen cargas de cuidado y atención es bajo: un $77,3 \%$ de las mujeres dice no tener cargas, frente al $89,3 \%$ de hombres.

Tabla 9. Cuidado y atención a personas dependientes

\begin{tabular}{|l|l|l|l|l|}
\hline \multirow{2}{*}{ Fuente de información } & Mujeres & Hombres \\
\cline { 2 - 6 } & Total & $\%$ & Total & $\%$ \\
\hline No & 34 & 77.3 & 24 & 85.8 \\
\hline $\mathrm{Si}$ & 10 & 22.7 & 4 & 14.2 \\
\hline$-\quad$ Padres & 4 & 9.1 & 2 & 7.1 \\
\hline$-\quad$ Hijos & 4 & 9.1 & 2 & 7.1 \\
\hline$-\quad$ Otros & 2 & 4.5 & 0 & 0 \\
\hline Total & 44 & 100 & 28 & 100 \\
\hline
\end{tabular}

La vigencia de la división sexual del trabajo, incipiente en este colectivo, pero que se va haciendo cada vez más evidente a medida que avanza la edad, constituye uno de los elementos destacados por numerosos investigadores para justificar la pérdida de mujeres a la largo de la carrera (Alcañiz; Arranz; León).

Cuando preguntamos por el principal responsable del trabajo reproductivo en el hogar (Tabla 10), encontramos que en ambos casos, el principal 
responsable del trabajo se comparte entre uno/a mismo/a y los dos miembros de la pareja por igual. Aunque la representación es anecdótica, vale la pena hacer notar que un hombre ha contestado que la persona responsable de las tareas de cuidado y domésticas es su pareja.

Tabla 10. Responsabilidad hacia el trabajo reproductivo en el hogar

\begin{tabular}{|l|l|l|l|l|}
\hline \multirow{2}{*}{ Fuente de información } & Mujeres & \multicolumn{3}{l|}{ Hombres } \\
\cline { 2 - 5 } & Total & $\%$ & Total & $\%$ \\
\hline Uno mismo & 18 & 40.9 & 13 & 46.4 \\
\hline La pareja & 0 & 0 & 1 & 3.6 \\
\hline Todos por igual & 22 & 50 & 10 & 35.7 \\
\hline Toda la familia igual & 2 & 4.5 & 3 & 10.7 \\
\hline Otros & 2 & 4.5 & 1 & 3.6 \\
\hline Total & 44 & 100 & 28 & 100 \\
\hline
\end{tabular}

\subsection{Mantenimiento: expectativas de futuro}

En este bloque se han querido conocer cuáles son las perspectivas laborales del PIF, ya sean dentro o fuera del ámbito universitario. Igualmente se ha querido identificar los canales a través de los cuales el PIF obtiene información sobre las salidas laborales una vez terminado el doctorado, y cuál es la valoración de la información recibida.

En primer lugar, queremos conocer qué proyecciones laborales tiene el PIF (Tabla 11):
A. Continuar vinculado al ámbito universitario y hacer carrera académica en la URV.
B. Continuar vinculado al ámbito universitario y hacer carrera académica en otra universidad.
C. Desvincularse de la universidad y optar por otras opciones profesionales.
D. No sabe.
E. Otros

Tabla 11. Perspectivas laborales después del Doctorado

\begin{tabular}{|l|l|l|l|l|}
\hline \multirow{2}{*}{ Fuente de información } & \multicolumn{3}{l|}{ Mujeres } & \multicolumn{3}{l|}{ Hombres } \\
\cline { 2 - 5 } & Total & $\%$ & Total & $\%$ \\
\hline OPCIÓN A & 12 & 27.3 & 5 & 17.9 \\
\hline OPCIÓN B & 6 & 13.6 & 6 & 28.6 \\
\hline OPCIÓN C & 5 & 11.4 & 5 & 17.9 \\
\hline OPCIÓN D & 19 & 43.2 & 8 & 26.6 \\
\hline OPCIÓN E & 2 & 4.5 & 2 & 7.1 \\
\hline Total & 44 & 100 & 28 & 100 \\
\hline
\end{tabular}


Observamos una gran diferencia en las respuestas: mientras que las respuestas de los hombres están muy divididas entre las 4 primeras opciones, las mujeres contestan mayoritariamente que no saben qué harán cuando finalicen el doctorado. Parece que la preferencia de las mujeres es continuar vinculadas a la URV y hacer carrera académica, mientras que los hombres se decantan por hacerlo en una universidad distinta.

Respecto a la posibilidad de desarrollar la carrera académica en la URV (Figura 4), queremos conocer cómo valora el PIF esta situación. El PIF encuestado ha valorado las perspectivas laborales en la URV en una escala que va de 1 (muy malas) a 5 (muy buenas). EL resultado es que, en términos generales, el PIF ve las oportunidades laborales en la URV muy malas $(36,1 \%)$. Sólo un $5,6 \%$ lo valora como bueno y un $2,8 \%$ como muy bueno.

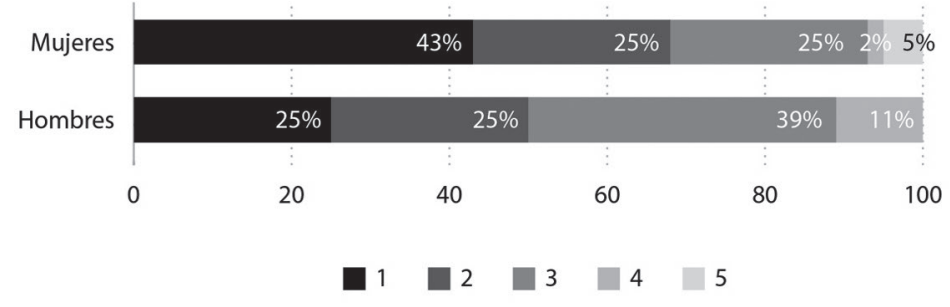

Figura 4. Valoración del futuro en la URV

Observamos, de nuevo, diferencias significativas entre mujeres y hombres. La mayoría de mujeres (un 43\%) ven su futuro en la URV muy mal, mientras que la mayoría de hombres (un 39\%) lo sitúan en el 3 de la escala de valoración. Es significativo contrastar esta pregunta con la anterior. Las mujeres apuestan preferiblemente para hacer carrera en la URV, pese consideran que las oportunidades son muy malas. Si en lugar de hacer el análisis de las perspectivas laborales por sexo, lo hacemos por área de conocimiento, vemos que el PIF más optimista respecto a su carrera en la URV es el de Ingeniería y Arquitectura, donde un 18,2\% considera que su futuro es bueno o muy bueno. Por otra parte, el PIF de Artes y Humanidades es el que peor valora su futuro en la URV: un $75 \%$ lo considera malo o muy malo.

En cuanto a la fuente de información de las salidas laborales, vemos que las mujeres reciben más información sobre salidas laborales que los hombres (52,3\% frente al 39,3\% de los hombres), tal como se muestra en la Figura 5. 


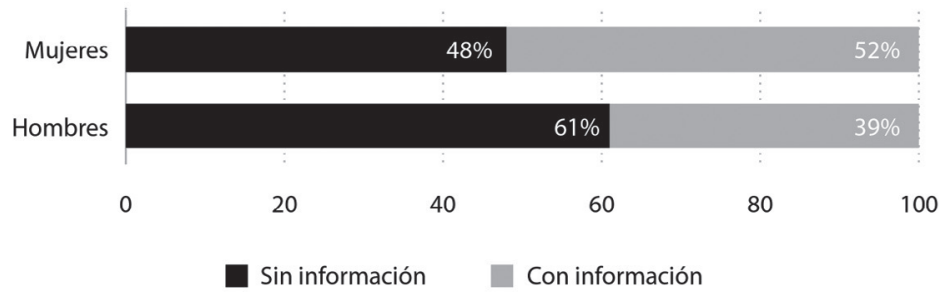

Figura 5. Reciben información adecuada

Sobre la procedencia de esta información (Tabla 12), vemos que para las mujeres un canal de comunicación relevante son los compañeros y compañeras de trabajo, mientras que los hombres reciben la información mayoritariamente a través de su director/a tesis. La segunda fuente de información relevante para las mujeres es el director/a de tesis, mientras que para los hombres, lo son las redes académicas y profesionales en el que están inscritos.

Tabla 12. Procedencia de la información sobre salidas laborales postdoctorales

\begin{tabular}{|l|l|l|l|l|}
\hline \multirow{2}{*}{ Fuente de información } & Mujeres & Hombres \\
\cline { 2 - 5 } & Total & $\%$ & Total & $\%$ \\
\hline Director de tesis & 15 & 34.1 & 9 & 32.1 \\
\hline Grupo de investigación & 10 & 22.7 & 2 & 7.1 \\
\hline EPD & 4 & 9.1 & 2 & 7.1 \\
\hline Otras instancias de la URV & 8 & 18.2 & 2 & 7.1 \\
\hline Compañeros/as de trabajo & 17 & 38.6 & 3 & 10.7 \\
\hline Redes académicas o profesionales & 10 & 22.7 & 4 & 14.3 \\
\hline Total & 44 & 100 & 28 & 100 \\
\hline
\end{tabular}

Todos los entrevistados coinciden en sus expectativas de hacer carrera académica, ya sea dentro o fuera de la URV. Es común en las entrevistas, independientemente del sexo y del ámbito de conocimiento, el reconocimiento de las dificultades que existen para poderse mantener en la carrera académica. El objetivo último, en todos los casos, es el de poder estabilizarse y ocupar un lugar permanente en el mundo académico. En términos generales, tal como muestran los resultados de la encuesta, la percepción de las posibilidades laborales dentro del ámbito universitario y, en concreto en la URV, son malas o muy malas. A partir de las entrevistas vemos cómo a medida que el PIF va conociendo mejor la dinámica universitaria, es más consciente de las dificultades de la carrera académica. Así, el PIF del primer año de beca se muestra más optimista que el PIF de los últimos años de beca. Además, aparece un factor 
determinante en la valoración del futuro académico, que es la relación con el grupo del departamento.

Tanto hombres como mujeres, en sus prospectivas para el futuro, visualizan un escenario difícil. Ambos evocan a la cuestión de la beca postdoctoral como un requisito para la estabilización en la Universidad. Para algunos es un tema prioritario tras doctorarse, mientras que para otros es un tema presente pero aún no resuelto. Vemos que los hombres se sitúan más en la primera opción, mientras que ellas muestran más dudas, motivados por el hecho de tener pareja y/o familia, tal como se ilustra en los siguientes fragmentos:

Me gustaría hacer una postdoc en la URV pero que pudiera ser flexible (...) Mi intención es tener una postdoc aquí pero flexible para estar en otro sitio. Mi pareja es de Austria (...) Me gustaría vivir en Viena, con mi pareja, porque él tiene empleo fijo. Por lo tanto la movilidad sería más bien mía hacia allá, que no al revés. (E. Mujer. 33 años)

El tema de la pareja sería algo a hablar. Él también es investigador. Está en la misma situación. A la hora de plantearme la movilidad postdoc, pues lo tendríamos que hablar. Dada la situación actual: iríamos donde la fuente de ingresos sea más fuerte, se priorizará eso. Porqué al final, no es que los dos vayamos a tener unos súper Trabajos, es que en el mejor de los casos uno de los dos tiene un trabajo normal. Y entonces ya verá el otro en que trabaja. (B. Mujer. 32 años)

Me gustaría hacer una postdoc en Cataluña, para no estar muy lejos de la URV (...) Además, vivo con mi pareja. Por ejemplo en la UB. Quizás estancias cortas más a menudo que no una estancia larga de 2 años. (F. Mujer. 25 años)

Por temas familiares, no me planteo una postdoc larga fuera de aquí, tal vez en las universidades de Barcelona o Lleida sí. (G. Mujer. 42 años)

Si bien la estancia postdoctoral se identifica como algo importante en la carrera académica, cuando pedimos que identifiquen otros factores importantes, todos coinciden en publicar con impacto (en dos entrevistas se repite la máxima del «publish or perish» de la carrera académica) y presentar comunicaciones a congresos. Sin embargo, algunos también reconocen la importancia de tener una buena red de contactos y buena relación con la universidad y el departamento. Así pues, para el PIF, el éxito en su carrera académica dependerá de 5 grandes factores: publicaciones, docencia, Estancias predoctorales, participación en congresos y red de contactos.

En cuanto a la información que el PIF recibe sobre las salidas profesionales una vez terminado el doctorado, las personas entrevistadas coinciden con los resultados de la encuesta en que las fuentes de las que se obtiene información son los compañeros y compañeras de trabajo y el director/a de tesis. Con todo, 
hay una idea generalizada de que si quieres saber información, debes ser autónomo para buscarte tú mismo las oportunidades.

\section{CONCLUSIONES}

Es un hecho constatado la progresiva desaparición de las mujeres de la carrera académica a medida que nos desplazamos hacia las categorías profesionales superiores. Lo que apuntan las estadísticas para el conjunto de universidades españolas y europeas se reproduce también en la URV. Como encarar esta realidad es el reto de las universidades para dejar de perder el talento femenino.

Tradicionalmente, el esfuerzo de las investigaciones en esta temática se ha centrado en identificar los factores externos a las mujeres, como la discriminación directa o indirecta, o los sesgos de género en los procesos de evaluación (Comisión Europea 2004). A pesar de estos factores tienen importancia, es necesario profundizar en los factores internos, es decir, analizar el proceso de selección social diferencial de hombres y mujeres y reflexionar críticamente sobre el impacto de la Universidad en su función socializadora.

En este trabajo hemos presentado una descripción estadística de la plantilla del personal investigador en formación de la URV, para dimensionar el fenómeno. Por otra parte, hemos presentado los resultados cuantitativos y cualitativos del trabajo de campo llevado a cabo. La idea principal del estudio que hemos expuesto es que si bien cada vez más hombres y mujeres comparten espacios en las primeras etapas como profesorado e investigadores de las universidades, se perciben diferencias en algunos aspectos de esta primera experiencia profesional dentro de la actividad académica. Diferencias que, mantenemos, no harán más que agrandarse a lo largo de los años de desarrollo profesional. Las diferencias encontradas en esta primera etapa se encuentran en aspectos como:

- El tipo de beca conseguida: hay una diferencia significativa entre mujeres y hombres, a favor de ellas, en la modalidad de becas FPI, FPU y AGAUR, convocatorias mucho más competitivas que las becas URV, donde se concentran mayoritariamente los hombres

- La vía de conocimiento de las becas: la mayoría de mujeres han conocido las becas a través de su director o directora de tesis, mientras que los hombres la han conocido a través de la universidad donde cursaron el máster. Es significativo que ninguna mujer ha conocido la beca a través del grupo de investigación, mientras que sí lo han hecho el $14,3 \%$ de los hombres. Este dato sustenta la hipótesis del mayor uso que hacen los hombres de las redes de contactos personales. También 
estaría mostrando las diferencias entre hombres y mujeres para acceder a la información.

- La carga docente: las mujeres hacen más docencia que los hombres e imparten más créditos. Esta tendencia suele extenderse a lo largo de la carrera académica, encontrando en el colectivo de mujeres una menor descarga de créditos asociados a los sexenios vivos, cargos de gestión y otras ventajas profesionales.

- También se dan diferencias en el tipo de hogar en el que viven: las mujeres viven mayoritariamente con su pareja mientras que los hombres viven mayoritariamente solos o con compañeros o compañeras de piso. En relación a la fuente principal de ingresos del hogar encontramos diferencias entre mujeres y hombres: los hombres muestran una mayor dependencia de los padres (14,3\% frente al $6,8 \%$ de las mujeres) y de su pareja (10,7\% frente al $6,8 \%$ de las mujeres). En todo caso, tanto para hombres como para mujeres, la fuente principal de ingresos del hogar es uno/a mismo/a. También se dan diferencias en el cuidado y la atención a personas dependientes ya que encontramos que hay más mujeres que hombres que asumen esta responsabilidad, si bien en ambos colectivos el porcentaje de personas que tienen cargas de cuidado y atención es bajo: un $77,3 \%$ de las mujeres dice no tener cargas, frente al $89,3 \%$ de hombres.

- El estudio realizado nos muestran también diferencias en aspectos muy relevantes por su impacto en la carrera profesional. En concreto el estudio muestra diferencias en cuanto a la fuente de información de las salidas laborales: las mujeres reciben más información sobre salidas laborales que los hombres.

A la luz de estas diferencias destacadas y como idea central del estudio que hemos llevado a cabo debemos decir que existe un consenso generalizado entre el PIF a favor de una cultura de la transparencia. Es de destacar que este es un discurso fundamentalmente de las mujeres. Son las mujeres las que protagonizan el discurso de la reivindicación de la claridad y transparencia de las «reglas de juego». Sin embargo, es imprescindible apuntar que la transparencia por sí sola no hará mejorar las posibilidades de promoción de las mujeres a no ser que, conjuntamente con la transparencia, se trabaje para asegurar que la Universidad como organización asume una política de erradicación de la arbitrariedad en los procesos.

La cuestión de los criterios a aplicar está estrechamente relacionada con la influencia del capital social en el desarrollo de la carrera profesional. Los 
comportamientos de networking 5 no tienen el mismo impacto sobre el desarrollo de la carrera académica de los hombres y mujeres en la creación de capital social. Se puede decir que estas acciones de networking se traducen en una ganancia de visibilidad interna y en una participación en actividades profesionales (que ayudan a la promoción profesional, participación en congresos, publicaciones, etc.). Estas diferencias podrían explicar las dificultades de acceso de las mujeres a toda una serie de recursos sociales principalmente controlados por los hombres. En este sentido, dominar o no las reglas del juego vinculadas a la práctica del ejercicio del poder es el eje alrededor del cual se articulan las diferencias de género.

Las mujeres difícilmente penetran en las redes profesionales y en los círculos de poder académico, en parte porque no han copiado las estrategias y ambiciones masculinas. Los obstáculos que se encuentran en el desarrollo de su trayectoria laboral las marginan y contribuyen a que ellas mismas en muchas ocasiones opten por renunciar, como una alternativa ante la dificultad para adherirse a los patrones masculinos de gestión con el que no se encuentran cómodos. Las mujeres en general, y las académicas en particular, no han conseguido «aculturalizarse» en un universo profesional dominado por unos códigos que no sienten como propios, ya que no han sido socializadas; podríamos decir que ellas son poco hábiles controlando las reglas no escritas implícitas en el ejercicio del poder.

A partir de los testimonios recogidos se ha observado que, bajo la apariencia de un sistema de promoción profesional formalmente igualitario, basado en las calificaciones (méritos) y la experiencia, aparecen unas limitaciones formales e informales en el desarrollo de la carrera profesional que configuran las expectativas y los horizontes profesionales de los becarios y las becarias.

Procurar la igualdad de género en el sistema universitario no es sólo una cuestión de justicia social (democrática) sino que, tal y como apunta el informe ETAN, es un indicativo de excelencia en la comunidad científica. Es por ello que la comunidad universitaria debería adoptar y utilizar estrategias y medidas concretas que eviten la discriminación por razón de género, especialmente para aquellas de carácter implícito (indirecto o inconsciente), alejándose de enfoques bien pensantes o benevolentes (hacer concesiones, de ayudar o que tratan como víctimas). La evaluación de la calidad en el entorno universitario debería pasar para controlar los sesgos de género que se producen en la

5. Ver: https://blogs.iadb.org/y-si-hablamos-de-igualdad/2015/04/23/por-que-los-hombresse-les-da-mejor-el-networking/ (Consultada el 10/07/2017) 
Academia, ya que la calidad de la ciencia pasa por desarrollar una investigación más sensible al género.

Debemos entender este trabajo como un primer diagnóstico, a partir de la cual desarrollar un plan de acción que nos permita modificar la situación desigual de hombres y mujeres que se constata en cada etapa de la carrera investigadora. Proponemos continuar este trabajo profundizando en, como mínimo, los siguientes tres aspectos que nos darían claves para la comprensión del fenómeno, como son:

- El impulso de la vocación científica de hombres y mujeres y su implicación con los estereotipos de género en el mundo de la ciencia.

- El impacto diferenciado de la paternidad y la maternidad y la conciliación de la vida académica con la vida familiar.

- Las barreras estructurales, formales e informales, a los que se enfrentan las mujeres a lo largo de la carrera académica, especialmente las que tienen lugar dentro de la propia universidad.

A la vez que hay que seguir haciendo investigación en torno a este fenómeno, hay que hacer visible el sexismo en la carrera académica entre la comunidad universitaria y hay que velar para que tanto el acceso como el desarrollo y la promoción de la carrera académica tengan lugar en un escenario de igualdad de oportunidades.

\section{REFERENCIAS BIBLIOGRÁFICAS}

Acker, Joan. «Hierarchies, Jobs, Bodies: A Theory of Gendered Organizations». Gender and Society, 2.4 (1990): 139-158.

Alcañiz, Mercedes. La conciliación de la vida laboral y familiar en la Universitat Jaume I. Castellón de la Plana: Publicaciones de la Universitat Jaume I, 2014. Arranz, Fátima. «Hombres y mujeres en el profesorado: un análisis de género en las académicas». En García de León, M. y García de Cortázar, M., Las académicas (profesorado universitario y género). Madrid: Instituto de la Mujer, 2001.

Barberá, Ester y Amparo Ramos. Mujeres directivas: promoción profesional en España y el Reino Unido. Vol. 2. Institut Universitari d'Estudis de la Dona, Universitat de València, 2000.

BOE (2007) Ley Orgánica 3/2007, de 22 de marzo, para la igualdad efectiva de mujeres y hombres, publicada en el BOE núm. 71, de 23 de marzo de 2007, páginas 12611 a 12645.

BOE (2007) Ley Orgánica 4/2007, de 12 de abril, por la que se modifica la Ley Orgánica 6/2001, de 21 de diciembre, de Universidades publicada en el BOE» núm. 89, de 13 de abril de 2007, páginas 16241 a 16260. 
BOE (2011) La Ley 14/2011 de la Ciencia, la Tecnología y la Innovación, publicada en el BOE num.131 de 2 de junio de 2011, páginas 54387 a 54455.

Comisión Europea. Gender and excellence in the making. Bruselas: Directorate General for Research Information and Communication Unit, 2004.

Ely, Robin J., y Debra E. Meyerson. «Theories of gender in organizations: A new approach to organizational analysis and change». Research in Organizational Behavior 22 (2000): 103-151.

ETAN. Política Científica de la Unión Europea. Promover la excelencia mediante la integración de la igualdad entre géneros. Bruselas: Comisión Europea, 2001.

Foschi, Martha. «Blocking the use of gender-based double standards for competence». En European Commission. Gender and Excellence in the Making, 51-56. Bruselas: Comisión Europea, 2004.

Generalitat de Catalunya. Doctes, doctores i catedràtiques. Cent anys d'accés lliure de la dona a la universitat. Barcelona: Consell Interuniversitari de Catalunya, 2010.

García de León, M. Antonia; Marisa García de Cortázar (Eds.). Las académicas (Profesorado universitario y género). Madrid: Instituto de la Mujer - MTAS, 2002.

Guil, Ana. «Techos universitarios de cristal blindado». Investigaciones Feministas 7.2 (2016): 25-39.

Izquierdo, María Jesús. El sexisme a la UAB. Propostes d'actuació i dades per a una diagnòstic. Bellaterra: Publicacions UAB, 2004.

Izquierdo, María Jesús. Cuidado y provisión: el sesgo de género en las prácticas universitarias y su impacto en la función socializadora de la universidad. Madrid: Ministerio de Igualdad, 2008.

Lara, Catalina. «La perspectiva de género en los sistemas de evaluación de la producción científica». Revista de Investigación Educativa 25.1 (2007):133-148.

León, Consuelo. «La mujer trabajadora y la nueva realidad laboral. El reto de armonizar trabajo y familia hoy». En Generalitat de Catalunya. Doctas, doctoras y catedráticas. Cien años de acceso libre de la mujer a la universidad. Barcelona: Consell Interuniversitari de Catalunya, 127-138, 2010

Ministerio de Educación, Cultura y Deporte. Datos y Cifras del sistema universitario español. Curso 2005-2006. Madrid: MECD, 2006.

Ministerio de Educación, Cultura y Deporte. Datos y Cifras del sistema universitario español. Curso 2014-2015. Madrid: MECD, 2015.

Méndez, Lourdes. «Administrando la desigualdad entre los sexos: ¿los estudios de género a la deriva?» En García Selgas, F. J. y Romero Bachiller, C. (Eds.), El doble filo de la navaja: Violencia y representación, Editorial Trotta. Madrid, 169-187, 2006.

Mora, Enrico. Diagnosi de la trajectòria acadèmica del personal becari de la UAB des de la perspectiva de gènere. Bellaterra: Publicacions UAB, 2006. 
Moss-Racusin, Corinne A., John F. Dovidio, Victoria L. Brescoll, Mark J. Graham, and Jo Handelsman. «Science faculty's subtle gender biases favor male students.» Proceedings of the National Academy of Sciences 109.41 (2012): 16474-16479

Nicolson, Paula. Poder, género y organizaciones: ¿Se valora a las mujeres en la empresa?, Madrid: Narcea Ediciones, 1997.

Pastor, Inma (Ed.). Cent anys de dones a la universitat. Tarragona: Publicaciones URV, 2011.

Pastor, Inma y Ana Acosta. «La institucionalización de las políticas de igualdad de género en la Universidad española. Avances y retos». Investigaciones Feministas 7.2 (2016): 247-271.

Pérez Sedeño, Eulalia, María Jesús Santesmases y Paloma Alcalá. Mujer y Ciencia. La situación de las mujeres investigadoras en el Sistema Español de Ciencia y tecnología. Madrid: Ministerio de Economía, Industria y Competitividad, 2007.

Sánchez de Madariaga, Inés (Coord.). Científicas en cifras. Madrid: Ministerio de Economía y Competitividad, 2014.

Sánchez de Madariaga, Inés, Sara de la Rica y Juan José Dolado. Libro Blanco. Situación de las mujeres en la ciencia española. Madrid: Ministerio de Ciencia e Innovación, 2011.

Wennerås, Christiney Agnes Wold. «Nepotism and Sexism in Peer-Review». Nature 387.22 (1997). 